\title{
Estudo de prevalência das fraturas buco-maxilo-faciais na região de Pelotas
}

\author{
Estudo de prevalência das fraturas buco-maxilo-faciais na região de Pelotas
}

\author{
Alex Teixeira Ferreira* \\ Moacir Portolan** \\ Marcos Antonio Torriani***
}

\begin{abstract}
RESUMO
Analisamos 745 casos de fraturas Buco-Maxilo-Faciais registrados no SAME (Serviço de Arquivo Médico Estatístico) da Santa Casa de Misericórdia de Pelotas e no Centro de Estudos e Reabilitação Buco-Maxilo-Facial de Pelotas (CERBMF), no período de 1 de janeiro de 1996 a 31 de dezembro de 2000. Os dados formam colhidos e anotados em fichas, as quais totalizaram 745 casos de pacientes submetidos à cirurgia. $O$ nariz foi o local mais atingido. As agressões foram as principais causas de fraturas $O$ gênero masculino foi o mais atingido, numa proporção de $8 / 2 \mathrm{em}$ relação ao feminino. A faixa etária mais atingida foi a de 21 a 30 anos de idade.
\end{abstract}

\section{PALAVRAS-CHAVE}

Fraturas Buco-Maxilo-Faciais; Traumatismo Buco-Maxilo-Faciais.

\section{INTRODUÇÃO}

Dentre as causas mais comuns de traumatismos Buco-Maxilo-Faciais que os autores têm proposto, podemos citar: agressões, quedas, acidentes automobilísticos, acidentes desportivos, maustratos às crianças, dentre outros. No entanto, são poucos os trabalhos que demonstram dados numéricos em relação à etiologia de fraturas Buco-MaxiloFaciais, bem como são poucos os dados epidemiológicos significativos. Desta forma, será necessário um maior controle de dados dentro de instituições e serviços que trabalham com esta área, para que se tenha condições de conhecer os reais números em relação ao trauma facial e para que se possa, a partir destes dados, iniciar um trabalho preventivo objetivando a formação de recursos humanos e de adequação dos serviços.

\section{REVISTA DA LITERATURA}

Uma análise realizada por Gerrie e Hakstian (1964) no Canadá, onde examinaram 1025 pacientes acima de 5 anos de idade, de 1958 a 1962, com fraturas dos ossos Maxilo-Faciais, mostram que os sítios mais freqüentes dessas fraturas são: ossos nasais (45\%), mandíbula (24\%), osso zigomático $(23 \%)$, maxila $(6 \%)$ e arco zigomático $(2 \%)$. Outros tipos de sítios não foram incluídos neste estudo. $O$ gênero masculino esteve presente em $77 \%$ dos casos, enquanto que, o feminino em $23 \%$ dos casos. As causas das fraturas maxilo-faciais foram: agressões em $30 \%$ dos casos; acidentes de trânsito em $24 \%$ dos casos; quedas em $20 \%$ dos casos; esportes $17 \%$ dos casos; acidente de trabalho em $4 \%$ dos casos; e outras etiologias em $5 \%$ dos casos.

Já Schultz (1967), constatou 478 casos de fraturas em ossos faciais. A média de idade dos pacientes foi de 32 anos e o gênero masculino predominou ligeiramente sobre o feminino. Os ossos próprios do nariz tiveram maior incidência nos casos, com um terço das fraturas. $O$ osso zigomático foi o segundo osso mais fraturado. As fraturas da maxila e alvéolodentárias representam os números próximos entre si, e aproximadamente com a mesma freqüência. A distribuição numérica é a seguinte: ossos do nariz 153, osso zigomático e arco zigomático 71 , maxila 48 e alvélo-dentária 46.

Rowe (1970) faz relato de seu trabalho de 1958, no Hospital Rooksdown House, Inglaterra, de 500 casos de fraturas dos ossos faciais em que 367 foram do gênero masculino e 113 do feminino. Em 112 pacientes havia a associação de fratura da maxila e outros ossos da face. A casuística das fraturas dos ossos do terço médio da face, totalizou 164 casos, distribuídos da seguinte forma: fraturas do zigomático 65 casos, arco zigomático 4 , pré-maxila 13, Le Fort II 14, Le Fort III
2, Le Fort I 31, Le Fort I e III 11 e Le Fort I, II e III 2 casos. As fraturas dos ossos do nariz associadas com fraturas de outros ossos faciais ocorreram em 75 casos. As alvéolo-dentárias somaram 82 casos. A maior incidência em relação a faixa etária ocorreu em pacientes entre 20 e 30 anos de idade.

Nakamura e Gross (1973) analisaram 323 pacientes com fraturas na face, na Universidade de Tennessee, de 1966 a 1970. A proporção entre homens e mulheres foi de 8:2. Os ossos atingidos foram: nariz, 28,80\%; mandíbula, 28,40\%; malar, 16,60\% ; maxilar, $12 \%$; arco zigomático, 8,50\%; Le Fort I, 3,70\%; blowout, $0,60 \%$; nasoetmoidofrontal, $1,4 \%$. As causas foram violência, $59 \%$; automóvel, $17 \%$; queda, 8, $10 \%$; esportes, $7,10 \%$; causas desconhecidas, $2,10 \%$; arma de fogo, $1,70 \%$; acidentes em indústrias, $1,40 \%$; outros veículos, $0,60 \%$ e outros, $2,90 \%$. Registraram, ainda, que, no ano de 1966, foram realizadas $128 \mathrm{ci}-$ rurgias de face sobre 5.997 cirurgias gerais, correspondendo a $2,13 \%$. No ano de 1967, o número total de cirurgias foi de 7.215 gerais, sendo 132 de face, correspondendo a $1,83 \%$. Em 1968, tiveram 126 casos de cirurgias de face e 5.844 cirurgias gerais, representando $2,16 \%$. Em 1969 foram 123 cirurgias faciais sobre 6.190 cirurgias gerais, ou $1,99 \%$, e, em 1970 , foram 132 de face sobre $6.667 \mathrm{ge}-$ rais ou $1,98 \%$.

$$
\begin{array}{r}
\text { * aluno da Especialização em Implantodontia; Uningá - PR. } \\
\text { ** Ms em CTBMF pela UFPel - RS. } \\
\text { *** Dr em CTBMF pela PUC - RS. }
\end{array}
$$


Mainous et al. (1974), no Hospital Naval de Long Beach, durante dois anos, estudaram fraturas Maxilo-Faciais. Foram 169 sítios em 118 pacientes. A maioria foi no gênero masculino, em pacientes jovens. A mandíbula atingiu 136 pacientes e o esqueleto fixo da face 32. Destas últimas encontraram: 18 pacientes com Le Fort I, 7 pacientes com fraturas de zigomático, 3 pacientes com Le Fort III, 2 pacientes com fraturas alveolares, 2 pacientes com Le Fort II. A etiologia mais comum foi a agressão, seguida dos acidentes de trânsito, quedas, esportes e outras.

No Departamento de Otrorrinolaringologia do Hospital Universitário de Lund, Sweden, na Suécia, Afzelius e Rosen (1980), avaliaram 368 pacientes hospitalizados com fraturas Maxilo-Faciais, e verificaram que a idade desses pacientes variava entre 4 e 92 anos de idade e que a idade média estava entre 20 e 24 anos. As vítimas de acidentes de trânsito, $81,3 \%$ dos pacientes, apresentavam fraturas do terço médio da face combinados, e $73,6 \%$ com fraturas Le Fort I e III. O sítio mais comum de fratura foi a do complexo zigomático-maxilar com 202 casos, seguidas pelas fraturas de mandíbula com 121 casos. A etiologia das fraturas difere em cada país, mas geralmente como maior causador, o trânsito está em primeiro lugar.

Montalvo (1989) relata que as prevalências das fraturas do esqueleto fixo da face atingiram os valores seguintes: nariz, 374 (61,01\%); alveolodentárias, 104 (16,97\%); zigomático, 67 (10,93\%); Le Fort III, $37(6,04 \%)$; Le Fort II, 20 $(3,26 \%)$; Le Fort I, sete $(1,14 \%)$; Le Fort I-II-III, uma $(0,16 \%)$. As fraturas tiveram predominância na faixa etária compreendida entre 20 a 29 anos $(24,59 \%)$. A prevalência do gênero masculino foi maior, chagando a $68,44 \%$, contra $31,56 \%$ do gênero feminino. As causas e as freqüências assinaladas foram: quedas, 68 $(32,07 \%)$; acidentes de trânsito, 60 $(28,30 \%)$; agressões, $55(25,94 \%)$; outras, $29(13,68 \%)$.

Díaz Jumbo (1990), em estudo realizado na zona sul do estado do Rio Grande do Sul, em 976 pacientes fraturados na face encontrou: ossos próprios do nariz, 505 casos $(51,74 \%)$; alveolodentária 145 (14,86\%); mandíbula, $122(12,50 \%)$; osso zigomático, $94(9,63 \%)$; Le Fort III, 59 $(6,05 \%)$, Le Fort II, 29 (2,97\%), Le Fort I, $13(1,33 \%)$; arco zigomático, nove $(0,92 \%)$. As fraturas dos ossos da face foram mais comuns nos homens, com 692 casos $(70,90 \%)$, contra 284 casos $(29,12 \%)$ nas mulheres. A etiologia não foi observada neste estudo.

Campos (1991), na região de Pelotas/ RS, Brasil, num estudo do ano de 1988 a 1989, demonstrou que, os 427 pacientes que deram entrada no Pronto Socorro da Fundação de Apoio Universitário (Pelotas), sofriam das mais variadas injúrias bucomaxilofaciais. Observou que, em relação às injúrias, 138 casos $(28,16 \%)$ ocorreram no nariz, $80(16,33 \%)$ no osso zigomático e 50 casos $(10,20 \%)$ na mandíbula. A agressão foi a maior responsável pelos traumatismos faciais, totalizando $37,46 \%$ dos casos. 0 gênero masculino somou 286 casos $(58,37 \%)$ e o feminino 204 (41,63\%). A faixa etária não foi pesquisada. Segundo o autor, há uma carência de dados informativos essenciais a uma pesquisa epidemiológica.

Barros e Souza (1993) estudaram 2250 casos de traumatismos bucomaxilofaciais, em pacientes de ambos os gêneros, relacionando-os com as causas das lesões, segundo a faixa etária. Observaram que $78,3 \%$ dos traumatizados foram do gênero masculino e $21,7 \%$, do feminino. Verificaram que a segunda, terceira e quarta décadas de vida foram as mais atingidas pelos acidentes e que $42 \%$ dos acidentes envolveram veículos automotores e $27 \%$, por distúrbios de comportamento (agressões).

Lobo (1993), analisando 3028 casos de fraturas bucomaxilofaciais, num período de seis anos (1987 a 1992), cujo atendimento foi realizado no Serviço de Cirurgia e Traumatologia Bucomaxilofacial do Hospital da Sociedade Beneficente de Campo Grande ( MS) conclui que: o osso zigomático foi o mais comprometido, com 726 casos de fraturas, seguido pela mandíbula com 445 casos; lesões em tecidos moles, com 383 casos e fraturas de nariz, com 344 casos. Os extremos encontrados, referentes à faixa etária, são de quatro a 68 anos de idade, sendo que, de 21 a 30 anos, foi o grupo etário mais atingido, seguido do grupo de 31 a 40 anos.

\section{PROPOSIÇÃO}

É proposta deste trabalho realizar um levantamento epidemiológico quantitativo de prevalência do traumatismo BucoMaxilo-Facial no Hospital da Santa Casa de Misericórdia de Pelotas (no Serviço de Arquivo Médico Estatístico e no Centro de Estudos e Reabilitação Buco-Maxilo-Facial) da cidade de Pelotas, durante o período de janeiro de 1996 a dezembro de 2000 .

\section{MATERIAL E MÉTODO}

Para a execução deste trabalho, foi realizada uma pesquisa junto aos prontuários do Centro de Estudos e Reabilitação Buco-Maxilo-Facial (CERBMF) do Hospital da Santa Casa de Misericórdia de Pelotas no período de janeiro de 1996 A dezembro de 2000 , na cidade de Pelotas/RS. Os dados colhidos, para este trabalho, foram anotados em fichas numeradas, especialmente elaboradas para este fim (Anexo 1), bem como utilizamos as anotações do Serviço de Arquivo Médico Estatístico (SAME) do Hospital da Santa Casa de Misericórdia de Pelotas/RS, que nos últimos cinco anos tem sido referência para o atendimento de pacientes com traumatismo buco-maxilo-faciais da região de Pelotas/RS.

Foram observados quatro fatores: etiologia das fraturas, sítio das fraturas, gênero e faixa etária. Em relação ao sítio das fraturas, consideramos os seguintes: nariz, osso zigomático, arco zigomático, mandíbula, Le Fort I, Le Fort II, Le Fort III, órbita, seio frontal e politraumas (quando ocorria em vários sítios). Considerando a etiologia, foi feito um apanhado das várias causas anotadas nos Registro da Santa Casa de Misericórdia de Pelotas/RS (SAME e CERBMF), sendo assim registrados: agressões, acidentes de trânsito, prática de esportes, quedas. Também foram considerados os gêneros: masculino e feminino; faixa etária, dividida da seguinte forma: 0 a 10 anos de idade, 11 a 20 anos de idade, 21 a 30 anos de idade, 31 a 40 anos de idade, 41 a 50 anos de idade, 51 a 60 anos de idade, 61 a 70 anos de idade e 70 anos de idade ou mais.

\section{RESULTADOS}

Baseados nos antecedentes e justificativas descritos no capítulo da revista da literatura, onde citamos o grande número de casos de traumatismo facial e, levando em conta que Pelotas faz parte de uma região com características específicas próprias, diferentes de qualquer outra região - aspectos socio-econômico-culturais, apresentaremos os dados em relação a etiologia, sítio de fratura, gênero e idade em que ocorrem as fraturas faciais, sob a forma de tabelas e gráficos como anteriormente colocado.

A Figura 1 apresenta a percentagem dos sítios das fraturas Buco-Maxilo-Faciais na região de Pelotas, no período de $1^{-}$de janeiro de 1996 a 31 de dezembro de 2000 , totalizando 745 casos.

A Figura 2 apresenta a prevalência das fraturas Buco-Maxilo-Faciais na região de Pelotas, demonstrando a etiologia, no período de $1^{\underline{0}}$ de janeiro de 1996 a 31 de dezembro de 2000 , totalizando 745 
casos.

A Figura 3 apresenta a prevalência das fraturas Buco-Maxilo-Faciais na região de Pelotas, demonstrando o gênero, no período de $1^{\circ}$ de janeiro de 1996 a 31 de dezembro de 2000.

A Figura 4 apresenta a prevalência das fraturas Buco-Maxilo-Faciais na região de Pelotas, demonstrando a faixa etária, no período de $1^{\underline{0}}$ de janeiro de 1996 a 31 de dezembro de 2000 .

\section{DISCUSSÃO}

$\mathrm{Na}$ literatura encontramos referência de que o traumatismo Buco-MaxiloFacial atinge indiscriminadamente o ser humano, não importando gênero, etnia, condição social ou local em que vive, podendo ocorrer em qualquer idade $(2,9)$.

Quanto à localização das fraturas, neste trabalho encontramos: o sítio mais freqüente de fraturas dos ossos da face, foi o nariz $(35,84 \%)$, seguido pelo osso zigomático $(21,74 \%)$ e a mandíbula $(20,54 \%)$, que representaram $78,12 \%$ dos 745 pacientes relatados. Foram considerados politraumas, aqueles indivíduos que apresentavam mais de um osso envolvido, com exceção das Le Fort I, II e III.

Portanto, os ossos próprios nasais são os ossos mais afetados no estudo retrospectivo dos cinco anos (1996 a 2000), sendo citados também como primeiros por autores como Campos (1991), Montalvo (1989), Nakamura e Gross (1973) .

A maior causa que levou a fratura dos ossos faciais em nosso trabalho de revisão de cinco anos (1996 a 2000), foi a agressão(um terço dos casos ou $33,55 \%$ ), esta mesma causa ocupou o primeiro lugar nos trabalhos de Gerrie e Hakstian (1984), Mainous et al. (1974).

Em relação à etiologia, temos os seguintes dados percentuais: agressão $33,55 \%$, acidente de trânsito $26,04 \%$, quedas $23,62 \%$, acidentes desportivos $9,65 \%$, perfazendo um total de $92,86 \%$ de todas as causas observadas nos 745 casos compilados nos cinco anos (1996 a 2000).

Em relação ao gênero, a totalidade dos autores pesquisados e citados neste trabalho, relatou maior prevalência do masculino sobre o feminino. Gerrie \& Hakstian (1966), encontraram um predomínio do gênero masculino sobre o feminino, onde o primeiro representou $77 \%$ dos casos. Segundo Schultz (1967), há uma ligeira predominância de envolvimento masculino quando comparado com o feminino. Rowe et al.(1970), em seu trabalho avaliou 500 casos (sítios) de fraturas dos ossos faciais, onde 367 foram do gênero masculino e 113 do feminino. Nakamura e Gross(1973) encontraram uma proporção homem/mulher de 8:2.

Em nosso estudo, como observamos a maior prevalência de fraturas Buco-Maxilo-Faciais quanto as faixas etárias, é entre 11 a 40 anos de idade, representando como média nos cinco anos estudados, $73,35 \%$ dos indivíduos. E a faixa etária mais atingida foi a dos 21 a 30 anos, o que coincide com autores como Afzelius e Rosen (1980), Gerri e Hakstian (1966), Nakamura e Gross (1973), Rowe e Killey (1970).

\section{CONCLUSÕES}

Após a revista da literatura e mediante os dados obtidos, chegamos às seguintes conclusões:

1. a maior prevalência de fraturas Buco-Maxilo-Faciais na região de Pelotas, em relação ao sítio, em ordem decrescente foi: nariz, osso zigomático e mandíbula;

2. em relação à etiologia das fraturas Buco-Maxilo-Faciais, a mais freqüente foi agressão, seguida por acidentes de trânsito, quedas e acidentes desportivos;

3. houve uma prevalência do gênero masculino sobre o feminino, numa proporção de $8 / 2$;

4. a faixa etária mais atingida foi a de 21 a 30 anos de idade, seguida pela de 31 a 40 anos de idade e a de 11 a 20 anos de idade.

\section{ABSTRACT}

We analyzed 745 cases of Buco-Maxilo-facial fractures registered in SAME (Service of Statistical Medical File) of Saint House of Mercy of Pelotas and in the Center of Studies and Buco-Maxilofacial Rehabilitation of Pelotas (CERBMF), in the period of january 1, 1996 to December 31, 2000. The data were selected and scored in records, which totalized 745 cases of patients submitted to the surgery. The nose was the most affected local. The aggressions were the main causes of fractures. The male gender was, in a proportion of $8: 2$ in relation to the females. The age group ranged from 21 to 30 years of age.

\section{KEYWORDS}

Buco-maxilo-facial fractures. BucoMaxilo-faciais traumatism.

\section{REFERÊNCIAS}

AFZELIUS, L.E. ; ROSÉN, C. Facial

Fractures: A Review of 368 Cases. Int. J. Oral. Surg., Copenhagen, v.9, p.2532,1980 .

BARROS, J.J.; SOUZA, L.C.M. Traumatismo Buco-Maxilo-Facial. São Paulo: Roca, 1993.
CAMPOS, P.D. Proposta para a Padronização de um Prontuário Específico para as Injúrias Bucomaxilofaciais. Pelotas. 1991. 69 f. Dissertação (Mestrado). Faculdade de Odontologia - Universidade Federal de Pelotas.

DÍAZ JUMBO, M. J. Ocorrência de Fraturas Bucomaxilofaciais: Contribuição a um Estudo Epidemiológico. Pelotas, 63f. 1990. Dissertação (Mestrado)- Ciências Faculdade de Odontologia. Universidade Federal de Pelotas.

GERRIE, J.W.; HAKSTIAN, R.W. Fractures of Facial Bones. Can Med Assoc J Ottawa, v. 90, p. 623-629, Mar. 1964.

LOBO, A.R. Incidência das Fraturas Bucomaxilofaciais na Região de Mato Grosso do Sul. Pelotas. 1993. 45f. Dissertação (Mestrado) - Faculdade de Odontologia. Universidade Federal de Pelotas.

MAINOUS, E.G. and cols. A Review of 168 Maxillofacial Fractures Treated at Naval Hospital. Mil Med, Washington, v. 80, p.139-278, Apr. 1974.

MONTALVO, A.A.M. Fraturas dos Ossos do Esqueleto Fixo da Face: contribuição a um estudo epidemiológico. Pelotas, 1989, 64f. Dissertação (Mestrado). Faculdade de Odontologia. Universidade Federal de Pelotas.

NAKAMURA, T.; GROSS, C.W. Facial Fractures: Analysis of Five Years of Experience. Arch Otolaryngol, Chicago, v. 97, p.288-290, Mar. 1973.

ROWE, N.L. Fractures of the Facial Skeleton in Children. J Oral Surg, Chicago, v.26, p. 505-515, Aug. 1968.

SCHULTZ, R.C. Facial Injuries from Automobile Accidents: A Study of 400 Consecutive Cases. Plast. Reconst. Surg., Baltimore, v.5, n.40, p.415425, Nov. 1967.

Recebido: 23 de março/2004

Aceito: 6 de dezembro/2004

Endereço para correspondência: Alex Teixeira Ferreira

Rua Dr. João Pessoa,

361 APT $^{\circ} 201-\mathrm{B}$

CEP: 96010470 Pelotas - RS

E-mail: atfrs@yahoo.com.br 


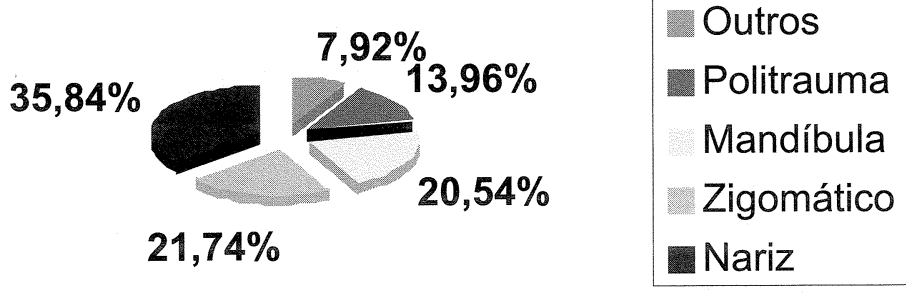

FIGURA 1. Distribuição das fraturas de acordo com o sítio (Pelotas - RS, 1996 a 2000).

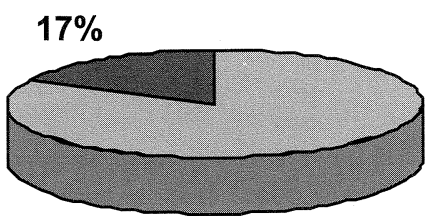

$83 \%$

FIGURA 3. Distribuição das fraturas de acordo com o gênero dos pacientes (Pelotas - RS, 1996 a 2000).

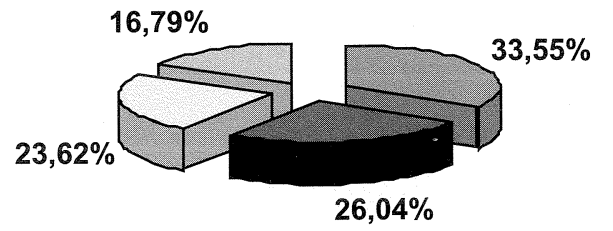

$\square$ Agressão

Acid. Trans.

$\square$ Queda

$\square$ Outros

FIGURA 2. Distribuição das fraturas de acordo com a etiologia (Pelotas - RS, 1996 a 2000).

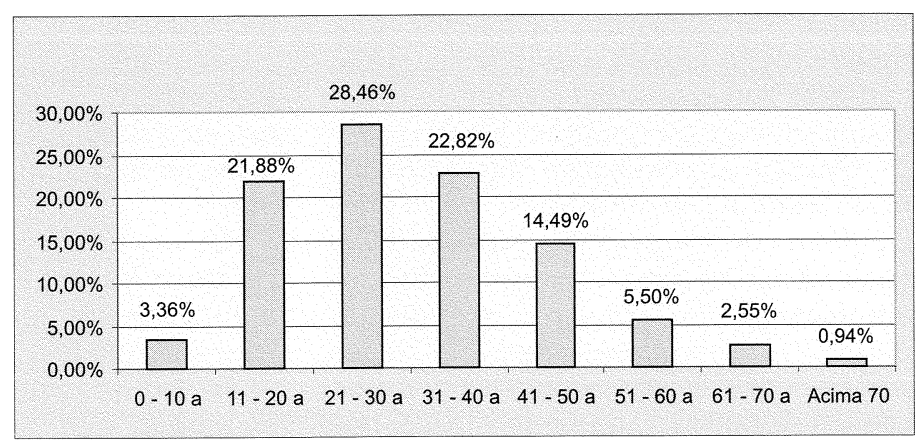

FIGURA 4. Distribuição das fraturas de acordo com a faixa etária dos pacientes (Pelotas - RS, 1996 a 2000). 